\title{
High CAMP in spores of Dictyostelium discoideum : association with spore dormancy and inhibition of germination
}

\author{
Kiran J. Virdy, ${ }^{1}$ Todd W. Sands, ${ }^{1}$ Susan H. Kopko, ${ }^{1}$ Saskia van Es, ${ }^{2}$ \\ Marcel Meima, ${ }^{2}$ Pauline Schaap ${ }^{2}$ and David A. Cotter ${ }^{1}$ \\ Author for correspondence: David A. Cotter. Tel: +15192533000 . Fax : + 15199713609. \\ e-mail : cotterl@uwindsor.ca
}

1 Department of Biological Sciences, University of Windsor, 401 Sunset

Avenue, Windsor, Ontario, Canada N9B 3P4

2 Institute of Molecular Plant Sciences, Cell Biology Section, Clusius Laboratory,

Wassenaarseweg 64, 2333

AL Leiden, Netherlands

\begin{abstract}
Signalling mechanisms involving CAMP have a well-documented role in the coordination of multicellular development and differentiation leading to spore formation in the social amoeba, Dictyostelium discoideum. The involvement of CAMP in the poorly understood developmental stages of spore dormancy and germination have been investigated in this study. Dormant spores contained up to 11-fold more CAMP than nascent amoebae. The spore CAMP levels were not constant, but typically underwent a surge at 14-18 $d$ when spores acquired the ability to germinate spontaneously. The high cAMP levels decreased only during successful spore germination, i.e. emergence of nascent amoebae. The temporal pattern of CAMP decrease was complex and unique to the method of spore activation, supporting our hypothesis that exogenously (e.g. heat) activated and autoactivated spores germinate by different mechanisms. During heat-induced activation, transcription of acg (a gene encoding adenylyl cyclase associated with germination) correlated well with spore CAMP content. Young wild-type spores, incapable of spontaneous germination, maintained a uniformly high CAMP level, and spore CAMP levels also remained high if germination was inhibited. When activated spores were deactivated by applying increased osmotic pressure, CAMP concentrations rose and ultimately levelled off at the high levels typical of dormant spores. The correlation between high CAMP and failure to germinate was also evident when autoactivation was inhibited by the CAMP analogue, 8-bromo-CAMP. Also, spores from a strain (HTY217) with unrestrained protein kinase $A$ activity were incapable of spontaneous germination. Overall, our experiments provide evidence for continued CAMP signalling in spores up to $18 \mathrm{~d}$ after sporulation and for linkages between elevated CAMP, spore deactivation and inhibition of spontaneous germination.
\end{abstract}

Keywords: Dictyostelium discoideum, cAMP, spore germination, spore dormancy

\section{INTRODUCTION}

Dictyostelium discoideum is an ideal model system for studying evolutionarily conserved signalling pathways. Its life cycle is well-defined and relatively simple: amoebae ultimately differentiate into one of two main cell types, spores or stalk cells. The developmental programme is exquisitely regulated by a number of signal molecules (see Loomis, 1982; Schaap, 1991;

Abbreviations: ACA, adenylylcyclase for aggregation; ACG, adenylylcyclase regulating spore germination; PKA, protein kinase $A$.
Cotter et al., 1992), including cAMP (see Van Haastert et al., 1991).

During terminal differentiation, elevated internal cAMP triggers spore formation (Kay, 1989; Riley et al., 1989; Riley \& Barclay, 1990; Maeda, 1992; Simon et al., 1992). The prespore-to-spore differentiation pathway is associated with sustained, non-pulsatile, high internal cAMP production (Peters et al., 1991), and a 3- to 4-fold higher internal cAMP concentration is required for expression of prespore than prestalk genes (Kwong \& Weeks, 1990). To generate such high levels, adenylyl cyclase activity is elevated during sporulation (Anjard $e t$ 
al., 1992). Transcripts for two distinct adenylyl cyclases, one for aggregation (aca) and the other for germination $(\mathrm{acg})$ are present in spores of young fruiting bodies (Pitt et al., 1992). Ammonia triggers spore formation by stimulating the accumulation and maintenance of high internal cAMP levels (Riley \& Barclay, 1990). Intracellular cAMP signalling and cell differentiation is mediated by protein kinase A (PKA) (Wang \& Kuspa, 1997; Firtel, 1996). 8-Bromo-cAMP activates PKA directly, and can trigger the differentiation of wild-type cells into spore and stalk cells (Maeda, 1992; Kay, 1989). The rapidly developing and sporogenous mutants $\operatorname{reg} A$ (Shaulsky et al., 1996; Loomis et al., 1997), rdeA (Kessin, 1977; Abe \& Yanagisawa, 1983) and ( $r d e C)$ HTY217 (Simon et al., 1992) exhibit elevated cAMP and/or PKA activity. As a nucleotide phosphodiesterase, RegA negatively regulates spore formation (Shaulsky et al., 1996; Loomis et al., 1997). Its inactivation allows cAMP accumulation to the high levels required for spore and stalk-cell development (Houslay \& Milligan, 1997; Thomason et al., 1998). The rdeA product, an H2 type phosphotransferase, participates in a multistep phosphorelay controlling RegA activity (Chang et al., 1998). Inactivation of RdeA elevates cAMP levels (Chang et al., 1998; Thomason et al., 1998).

Although cAMP and associated signalling pathways leading up to and including sporulation have been investigated in depth, the signalling mechanisms governing spore dormancy and germination are largely unknown. Spore germination is a complex process involving removal of the spore matrix containing an autoinhibitor (Abe et al., 1976), and can be externally induced by imposing physical/chemical treatments such as heat shock (Dahlberg \& Cotter, 1978; Cotter, 1981), or is induced spontaneously through autoactivation (Dahlberg \& Cotter, 1978; Cotter \& Glaves, 1989). During autoactivation, spores secrete a low-molecularmass phosphorylated adenine derivative (autoactivator) that is neither cAMP nor cGMP (Dahlberg \& Cotter, 1978; Glaves \& Cotter, 1989; Cotter et al., 1990).

Externally activated spores germinate rapidly and synchronously; however, they do not synthesize, secrete or respond to autoactivator (Cotter \& Glaves, 1989). Young (1-3 d old) wild-type NC4 spores cannot autoactivate, and neither produce nor respond to the autoactivator (Dahlberg \& Cotter, 1978). In contrast, aged (at least $8 \mathrm{~d}$ old) wild-type spores gradually acquire the ability to respond, and after 10-14 $\mathrm{d}$ in the sorocarp, strongly respond to and autonomously produce this factor (Dahlberg \& Cotter, 1978). Spontaneous germination in aged NC4 spores begins only after a 5-8 h lag; addition of autoactivator considerably shortens both the lag and overall germination time (Dahlberg \& Cotter, 1978; Cotter \& Glaves, 1989). After spore activation, germination is divided into three major stages: post-activation lag, spore swelling and emergence of amoebae (Cotter, 1981). Spores are extremely sensitive to the environment during early germination. In adverse conditions such as high osmotic pressure or reduced aeration, activated spores deactivate at the end of the lag phase and remain dormant even if the deactivating conditions are reversed (Cotter, 1981).

Recent studies have suggested a role for cAMP in spores of $D$. discoideum (Cotter et al., 1992; van Es et al., 1996). ACG has been identified as an osmosensor regulating spore dormancy and germination (van Es et al., 1996). Preliminary studies have indicated that agents antagonizing cAMP signalling (caffeine or adenosine) temporally accelerate germination, whereas agonists (dibutyryl-cAMP) delay or inhibit it (Cotter et al., 1992). To clarify the role of cAMP we have examined spores during dormancy and germination.

\section{METHODS}

Organism and germination. In most experiments, wild-type D. discoideum strain NC4 (ATCC 24697) was used. For Northern blotting, acg mRNA was from spores and amoebae of strain AX3. The mutant D. discoideum strain HTY217, generously provided by Dr M. Veron (The Pasteur Institute, Paris), was studied for germination phenotype. D. discoideum strains were grown with Escherichia coli B/r (ATCC 12407)) on glucose-salts agar in Petri plates (Cotter \& Raper, 1968), and after spore formation intact fruiting bodies were allowed to age at $21-23^{\circ} \mathrm{C}$. Spores were harvested by shaving spore heads from the fruiting bodies with the edge of a glass slide; spore heads were suspended in $10 \mathrm{mM} \mathrm{KH}_{2} \mathrm{PO}_{4}$ germination buffer $(\mathrm{pH} \mathrm{6.5)}$ and centrifuged at $4500 \mathrm{~g}$ for $3 \mathrm{~min}$. Supernatants containing autoinhibitor (Abe et al., 1976) were decanted and spore pellets were washed three times in germination buffer (Cotter \& Raper, 1968).

For autoactivation, appropriately aged spores were harvested, washed as above and resuspended in germination buffer, or in germination buffer containing autoactivator (Dahlberg \& Cotter, 1978; Cotter \& Glaves, 1989). The effect of 8-bromocAMP was tested using buffer with $9 \mathrm{mM} 8$-bromo-cAMP and $1 \mathrm{mM} \mathrm{KH} \mathrm{PO}_{4}$ to maintain an osmotic pressure equivalent to $10 \mathrm{mM} \mathrm{KH} \mathrm{KO}_{4}$ germination buffer. Germination experiments were conducted at $21-23^{\circ} \mathrm{C}$. Phase-contrast microscopy was used to estimate the percentages of unswollen spores, swollen spores and nascent amoebae in germinating spore suspensions (Cotter, 1981).

Sample preparation and CAMP determinations. To estimate internal cAMP levels, spores were washed (freshly harvested spores) or simply collected (germinating spore suspensions) and centrifuged at $4500 \mathrm{~g}$ for $3 \mathrm{~min}$ (Cotter \& Raper, 1968). Total spore densities were $3-6 \times 10^{8}$ spores $\mathrm{ml}^{-1}$. Spores or cells were mechanically lysed in ice-cold distilled water $(400 \mu \mathrm{l})$ containing $10 \mathrm{mM}$ DTT by vortexing with $0.25-0.32 \mathrm{~mm}$ diameter acid-washed glass beads for 1.5-2 min (amoebae) or 12-15 min (spores) (North \& Cotter, 1984). The efficiency of breakage was determined microscopically (North \& Cotter, 1984). Extracts were deproteinized (Goldberg \& O'Toole, 1971 ) by adding $2.0 \mathrm{ml}$ ice-cold $6 \%(\mathrm{w} / \mathrm{v})$ trichloroacetic acid, incubating $15 \mathrm{~min}$ on ice, and centrifuging at $4500 \mathrm{~g}$ for $5 \mathrm{~min}$. Supernatants $(2.0 \mathrm{ml})$ clarified by an additional centrifugation and extracted four times with $10 \mathrm{ml}$ diethyl ether were dried for $2 \mathrm{~h}$ under nitrogen at $60^{\circ} \mathrm{C}$ and the residue was stored desiccated at $-20^{\circ} \mathrm{C}$ for up to $14 \mathrm{~d}$ with no detectable loss of cAMP.

cAMP in each sample was determined by dissolving the dried extract in $0.05 \mathrm{M}$ acetate buffer $\left(\mathrm{pH} \mathrm{5.8)}\right.$ and using a ${ }^{125} \mathrm{I}$ 
cAMP scintillation proximity radioimmunassay (Amersham). Acetylation of samples was not necessary. Each sample was counted for 2 min (Beckman LS 3801), and counts for two consecutive cycles were averaged and compared with standards by using a simple linear regression.

Measurements were obtained from at least three independent experiments involving different batches of similarly aged spores or cells. The reproducibility of data indicated that the trends observed in spore cAMP levels were not caused by sampling error, nor variation in the efficiency of breakage. Breakage times were adjusted to maintain high breakage efficiencies $(80-90 \%)$ for dormant as well as swollen spores: the breakage efficiency of amoebae samples was consistently $100 \%$. In additional control experiments, where spore samples were divided and a known concentration of cAMP was added to one-half prior to processing, the final cAMP levels did not differ by more than 2 pmol from expected values. DTT, an inhibitor of extracellular phosphodiesterase activity (Kessin $e t$ al., 1979), was added to all samples even though final cAMP results were identical to samples processed without this reducing agent. Bacteria produce detectable levels of cAMP (Botsford \& Harman, 1992); however, in spore samples cAMP contamination from this source was negligible because of the selective harvesting and washing procedures (Cotter $\&$ Raper, 1968).

Effect of heat shock on acg mRNA. Young ( $4 \mathrm{~d}$ old) AX3 spores were heat-shocked at $45^{\circ} \mathrm{C}$ for $30 \mathrm{~min}$ (time 0 ) and at intervals thereafter the spores/amoebae were collected and total RNA was isolated from $2 \cdot 5 \times 10^{7}$ spores as described by Nellen et al. (1987), except that after the first addition of phenol to the lysate, $0.1 \mathrm{~g}$ of glass beads (diameter $0.5 \mathrm{~mm}$ ) was added and samples were vortexed for $10 \mathrm{~min}$ to break the spore walls. Total RNA was size-fractionated on $1.5 \%$ agarose gels containing $2 \cdot 2 \mathrm{M}$ formaldehyde and transferred to nylon membranes. The RNA was then hybridized to a $\left[{ }^{32} \mathrm{P}\right] \mathrm{dATP}$-labelled acg probe by standard procedures (van Es et al., 1996) and exposed to X-ray films. The quality of loading was tested by electrophoresing duplicate RNA samples and staining with ethidium bromide.

\section{RESULTS}

\section{CAMP in ageing 'dormant' spores}

Asexual development is generally considered complete with the formation of spores, hence dormant spores were expected to be inert and demonstrate constant cAMP concentrations. However, wild-type spores analysed for cAMP content at early (1-2 d), middle (4-6 d) and late (14-18 d) stages after spore formation showed differences in cAMP content, with the 14-18 d sample at 32.9 pmol per $10^{8}$ spores containing the highest concentrations (Fig. 1). Regardless of age, the cAMP content of spores was consistently higher (up to 11-fold) than that in nascent amoebae. Therefore, we predicted that the decline to the low levels in amoebae occurred during spore germination.

\section{CAMP during spore germination}

We determined cAMP levels in autoactivated and heatactivated NC4 spores. In both, cAMP concentrations were consistently higher than those in nascent amoebae.

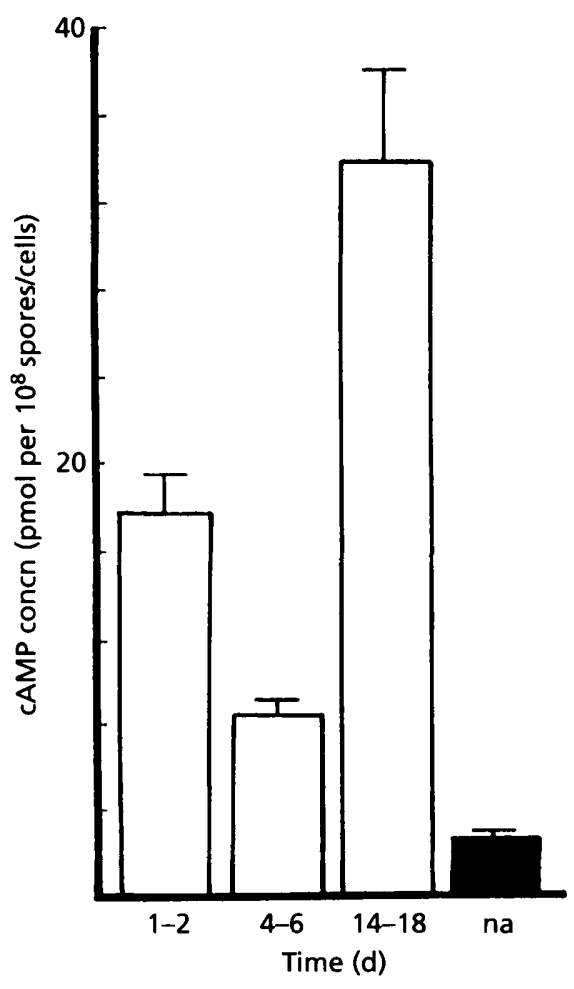

Fig. 1. Changing CAMP levels during spore 'dormancy'. NC4 spores were harvested after 1-2 d, 4-6 d and 14-18 $d$ of ageing in fruiting bodies, and analysed for CAMP content. Data are reported as mean and standard error for pmol CAMP per $10^{8}$ spores or cells in three independent determinations. na represents the CAMP level in five independent samples of newly emerged amoebae.

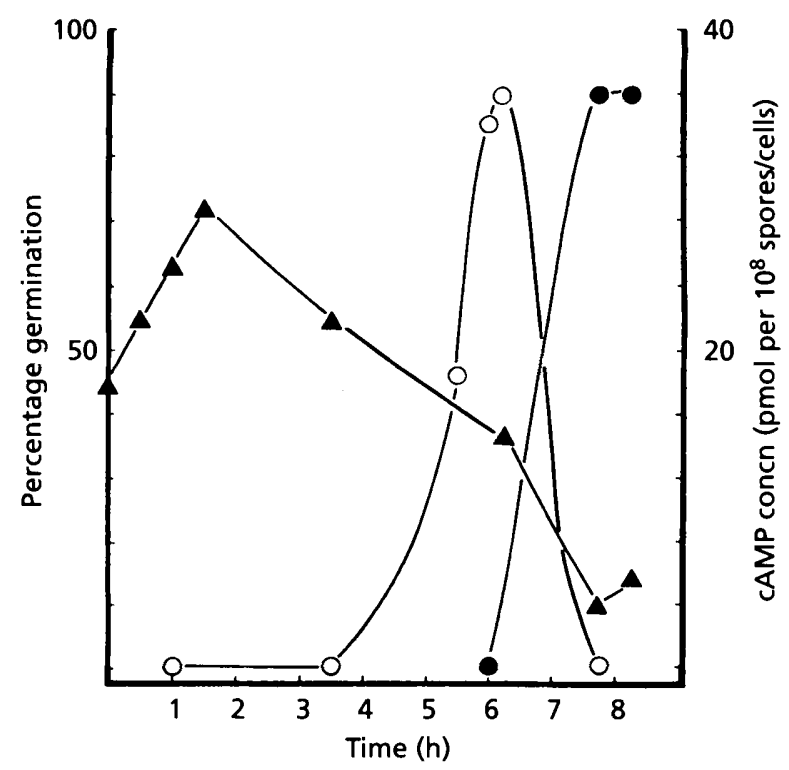

Fig. 2. Autoactivation kinetics of NC4 spores (13-15 d) at $2.9 \times 10^{8}$ spores $\mathrm{ml}^{-1}$. $O$, Percentage of swollen spores; percentage of nascent amoebae; $\Delta$, pmol CAMP per $10^{8}$ spores/cells. 


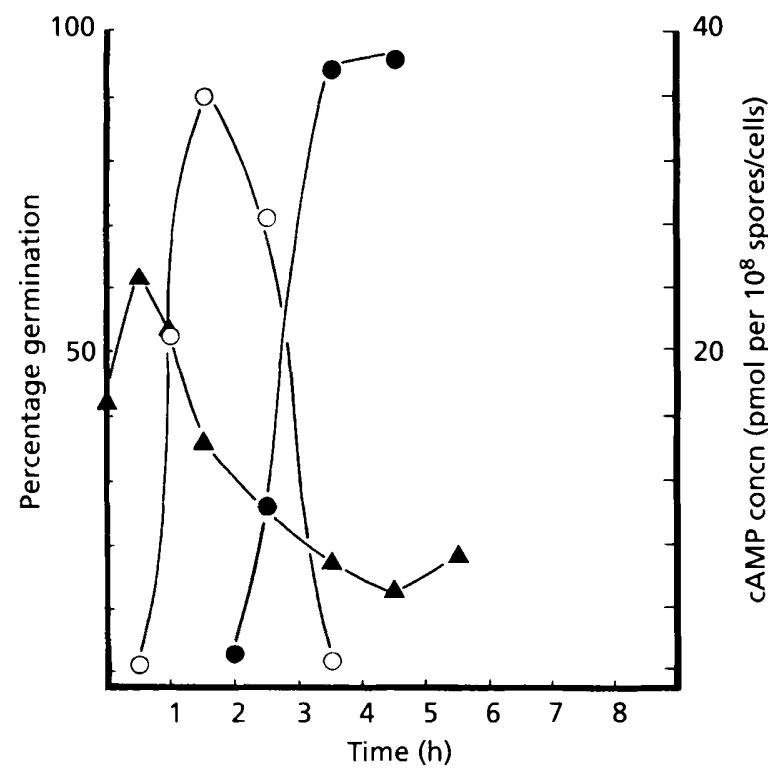

Fig. 3. Levels of CAMP during autoactivator-induced spontaneous germination. Autoactivator was added immediately after harvesting and washing of aged (13-15d) NC4 spores. Germination was monitored as the percentage of swollen spores $(O)$ and the percentage of nascent amoebae (O). Black triangles $(\boldsymbol{A})$ show pmol CAMP per $10^{8}$ spores/cells.

During autoactivation, cAMP levels increased by at least 10 pmol per $10^{*}$ spores in early germination, peaked during the lag phase, and then declined to the low levels detected in amoebae (Fig. 2). In contrast, young spores (incapable of autoactivation) maintained cAMP at the dormant spore level of $15-20$ pmol per $10^{8}$ spores throughout the $8 \mathrm{~h}$ of attempted autoactivation (data not shown). This indicates that the increase during early germination is not a generalized reaction of spores to being physically removed from the sorocarp milieu.

Addition of autoactivator to aged spores accelerated germination and shortened the lag time; spore cAMP increased by at least 8 pmol over the levels present just before swelling began (Fig. 3). Hence, the pattern of spore cAMP changes during autoactivation, typified by a surge and then a decline, was retained despite the altered lag time.

The cAMP pattern during germination of heat-activated spores was distinct from that for autoactivation. Immediately after heat shock, cAMP levels decreased to $1 / 3$ of that in similarly aged freshly harvested spores, then peaked during maximum spore swelling and finally decreased to levels in nascent amoebae (Fig. 4a). This pattern correlated with the timing of acg transcription (Fig. 5). The transcript was observed at time 0 , before heat-activation, and was virtually undetectable $30 \mathrm{~min}$ after heat shock, when the cAMP level had fallen. As germination ensued with spore swelling, the transcript was again generated. However, both the acg transcript and CAMP levels were significantly reduced with full

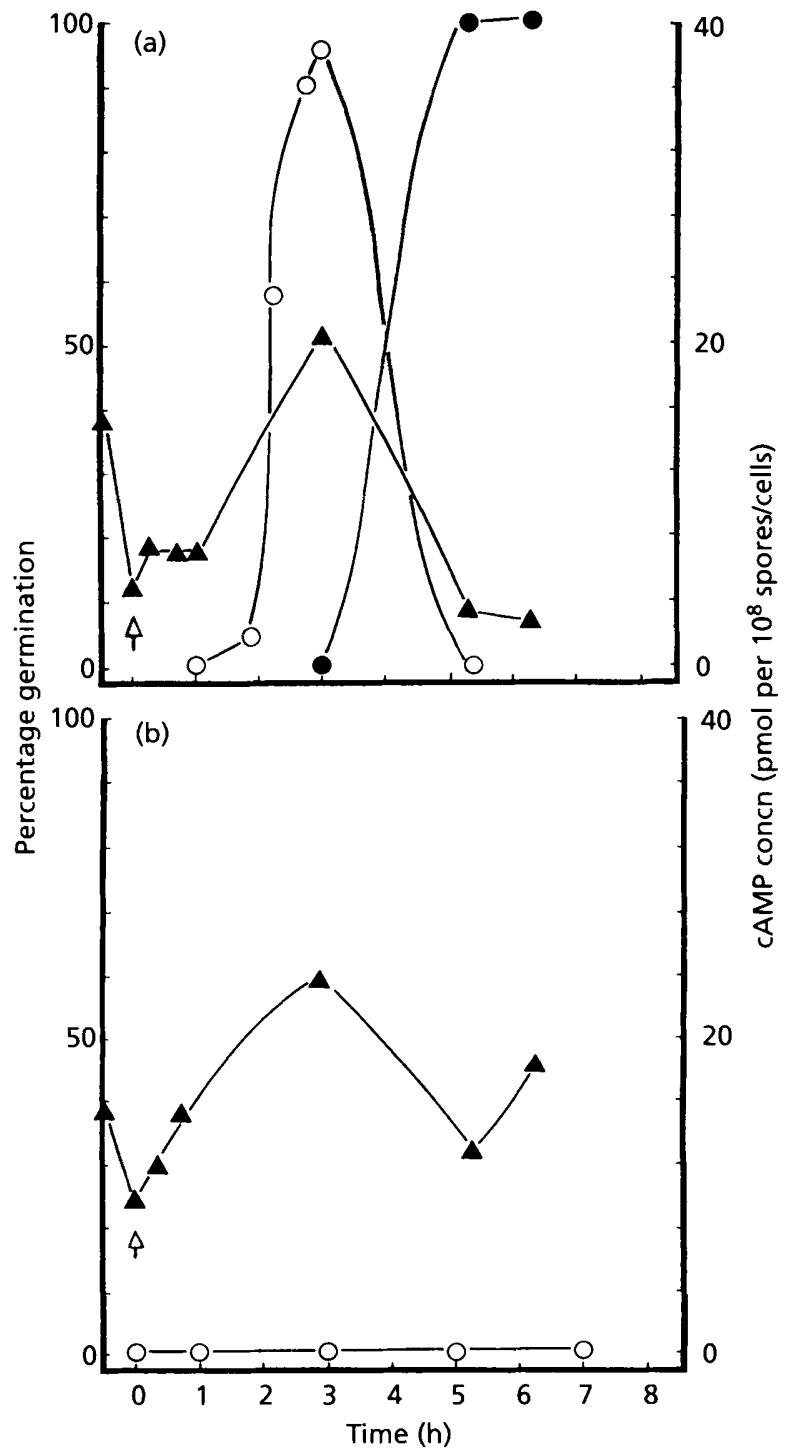

Fig. 4. Levels of CAMP during heat shock-induced germination and sucrose-induced deactivation of spores. (a) Heat-induced germination of NC4 spores (13-15 d) at $5.9 \times 10^{8}$ spores $\mathrm{ml}^{-1}$. (b) Spore deactivation of NC4 spores (13-15d) at $1.8 \times 10^{8}$ spores $\mathrm{ml}^{-1}$ in $0.25 \mathrm{M}$ sucrose. The arrow indicates termination of heat shock. Germination was monitored as the percentage of swollen spores $(O)$ and the percentage of nascent amoebae (๑). CAMP was determined as pmol per $10^{8}$ spores/cells $(\boldsymbol{\Delta})$.

emergence of the amoebae (Fig. 5). Hence, the disappearance of acg mRNA correlated well with the reduction in cAMP levels after heat shock.

cAMP levels during the first $3 \mathrm{~h}$ after sucrose-induced spore deactivation, resembled those in heat-activated spores (see Fig. 4). Collectively, these data suggest that the transient cAMP increase after heat activation is not strictly coupled to spore swelling but rather to early germination events during the lag phase. After the transient increase and subsequent decline, the cAMP level in deactivating spores returned to that of freshly 


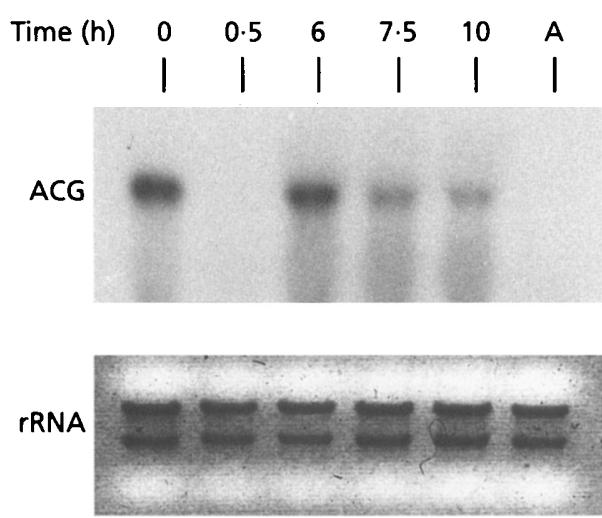

Fig. 5. acg mRNA levels during heat-induced germination. The following is a summary of the germination data. $t=0 \mathrm{~h}, 100 \%$ dormant spores; $\mathrm{t}=0.5 \mathrm{~h}$ after heat activation, $100 \%$ activated unswollen spores; $t=6 \mathrm{~h}, 83.3 \%$ swollen spores and $0.5 \%$ nascent amoebae; $t=7.5 \mathrm{~h}, 81.9 \%$ swollen spores and $15.4 \%$ nascent amoebae; $t=10 \mathrm{~h}, 4.1 \%$ swollen spores and $95 \%$ nascent amoebae; A, $100 \%$ nascent amoebae. Below the Northern hybridization blot is a negative image of the ethidium-bromide stained bands of RNA from the same samples, demonstrating equal loading at each sampling time.

harvested spores as dormancy was reacquired (see Fig. $4 \mathrm{~b})$. In general, the germination experiments showed high cAMP levels in spores and its dramatic decline only with the emergence of nascent amoebae.

\section{Effects of CAMP agonists on spore germination}

The permeant cAMP analogue 8-bromo-cAMP, which bypasses the surface cAMP receptor and activates PKA directly (Simon et al., 1992), inhibited autoactivation of aged $(10 \mathrm{~d})$ wild-type spores. Over $24 \mathrm{~h}$, germination occurred in less than $2 \%$ of spores treated with $9 \mathrm{mM}$ 8-bromo-cAMP in $1 \mathrm{mM} \mathrm{KH}_{2} \mathrm{PO}_{4}$ buffer whereas over $97 \%$ of untreated spores ( $10 \mathrm{mM} \mathrm{KH_{2 }} \mathrm{PO}_{4}$ buffer) germinated. The treated spores did not autoactivate during a further $24 \mathrm{~h}$ after removal of exogenous 8-bromo-cAMP by washing and resuspension in $10 \mathrm{mM}$ $\mathrm{KH}_{2} \mathrm{PO}_{4}$ buffer; however, the treated spores remained viable and germinated after heat-activation at $45^{\circ} \mathrm{C}$ for 30 min (data not shown).

Spores of strain HTY217 with unrestrained PKA activity (Simon et al., 1992) had only a limited ability to germinate spontaneously; less than $10 \%$ of the spores aged $10 \mathrm{~d}$ germinated over $24 \mathrm{~h}$.

\section{DISCUSSION}

Previous cAMP determinations in $D$. discoideum revealed 10 -fold higher cAMP concentrations in fruiting bodies than in vegetative cells (Malkinson \& Ashworth, 1973). However, the amount contributed by spores, stalk cells and matrix components was not defined, nor were the amounts within spores during germination estimated.
cAMP levels up to 11-fold higher in dormant spores than in nascent amoebae are attributed, in part, to the activity of ACG, the germination-specific adenylyl cyclase, (Pitt et al., 1992; van Es et al., 1996) and conceivably are sustained by high spore ATP content (Cotter et al., 1992; Klein et al., 1988). ACG, present only in spores (Pitt et al., 1992), promotes spore dormancy via elevated external osmolarity (van Es et al., 1996). Ammonium phosphate, found in the sorus at over $100 \mathrm{mM}$, is a major effector of this osmosensing pathway (Cotter $e t$ al., 1999). Ammonia may further promote internal cAMP accumulation by inhibiting cAMP secretion and degradation (Riley \& Barclay, 1990). Also contributing in this way to high cAMP concentrations is the inhibition of RegA cAMP phosphodiesterase activity (Shaulsky et al., 1998; Zinda et al., 1998). Therefore, spore formation and the maintenance of dormancy seem to require high internal cAMP levels generated by ACG (van Es et al., 1996) and decreased RegA activity (Shaulsky et al., 1998). Perhaps a similar mechanism is associated with the dormancy of other spore-like structures such as micro-like cysts and macrocysts (Cotter et al., 1992).

Sporulation has long been considered to herald the completion of development. However, the evidence for cAMP signalling throughout 'dormancy' suggests that development continues beyond spore formation. As spores age, their cAMP levels remain dynamic and always higher than those in amoebae. The peak level at 14-18 d coincides with the maximum ability of wildtype spores to germinate spontaneously. After this, they slowly lose viability, and hence surrender the opportunity to reinitiate the life cycle. Spore dormancy and viability up to $20 \mathrm{~d}$ also correlate with high actin tyrosine phosphorylation activity (Kishi et al., 1998). The meaning of the cAMP peak at $14-18 \mathrm{~d}$ is, as yet, unclear. Perhaps, a cAMP-dependent pathway activates "late spore-maturation genes' (Glaves \& Cotter, 1989), yielding products needed during late dormancy. Such products may be required for spore ageing/viability or autoactivation (Glaves \& Cotter, 1989), and/or actin tyrosine phosphorylation (Kishi et al., 1998). Alternatively, the changes in cAMP levels during spore ageing may simply reflect a changing osmotic environment transduced by the ACG osmosensor (van Es et al., 1996).

Spore germination represents the conversion of cells from a developmental to a vegetative state (Chandrasekhar et al., 1992). During this crucial stage, the elevated cAMP level in spores decreases to a negligible level only when nascent amoebae emerge. Previously, we had hypothesized that alterations in a cAMP cascade intrinsic to spores could trigger germination (Cotter et al., 1992). Therefore, in experiments where germination was inhibited (attempted autoactivation of young spores and sucrose-induced spore deactivation), we were not surprised when cAMP did not decrease to the levels in amoebae but stabilized at those in dormant spores. The association of high cAMP with spore dormancy was further strengthened by the inhibition of autoactivation by 8 -bromo-cAMP. The failure of spores treated with 8 -bromo-cAMP to ger- 
minate spontaneously after removal of the agonist may have been due to spore deactivation through a cAMP/ PKA-dependent pathway, since the deactivated spores, although unable to autoactivate, could be triggered to germinate with external activation (heat shock). It is noteworthy that another cAMP agonist, dibutyryl cAMP at $10 \mathrm{mM}$, inhibited autoactivation similarly (data not shown), but that neither agonist changed the germination kinetics of heat-activated spores (data not shown).

RegA in wild-type spores is presumed to lower cAMP and PKA to levels compatible with germination (Loomis, 1998). Hence, spores of the RegA mutant of $D$. discoideum germinate poorly (Shaulsky et al., 1998). In contrast, spores of the ACG null mutant, with approximately $50 \%$ of the cAMP content in the wild-type spores (data not shown), germinate well without heat shock activation and in high osmolarity (van Es et al., 1996). The presence of some cAMP in ACG null mutants suggests another source for cAMP (Kim et al., 1998).

Although acg transcription coincided approximately with $c A M P$ activity during heat-induced germination, the relationship between transcriptional control and cAMP production is unclear; acg transcription may be coupled to other germination signals besides osmotic pressure. ACG inhibits germination via PKA (van Es et al., 1996). D. discoideum mutants HTY 217 and Sp60PKA, both demonstrating high PKA activity, germinate very poorly (van Es et al., 1996). Recently, the twocomponent histidine kinase DHKB has been shown to be required for maintenance of spore dormancy and inhibition of germination, presumably via PKA (Zinda \& Singleton, 1998). Interestingly, $d h k B$-null spores, which contain $60 \%$ less intracellular cAMP than their wild-type parents, germinate precociously in situ (Zinda \& Singleton, 1998).

Assuming that germination is consistently associated with decreasing cAMP levels in both heat-induced germination and autoactivation, the cAMP increase during early germination is a paradox. In autoactivation, cAMP increases in the lag phase, during which spores are highly sensitive to environmental parameters. If external conditions become unfavourable, spores readily deactivate and reacquire dormancy (Cotter, 1981). During heat activation, the cAMP peak coincided with maximum spore swelling, and this CAMP pattern was preserved during sucrose-induced deactivation, where spores did not swell. If we assume that high cAMP is necessary for dormancy and that autoactivation is more physiologically relevant, then the increase in cAMP during early germination may represent a highly conserved pathway to ensure rapid spore deactivation and re-establishment of dormancy during adverse conditions.

The unique cAMP profiles and the different responses of heat-activated and autoactivated spores to various agents (Cotter et al., 1992), including cAMP agonists, support the view that the regulatory mechanisms governing these germination processes are distinct
(Dahlberg \& Cotter, 1978; Cotter \& Glaves, 1989). As further evidence, Lydan et al. (1994) have shown that the pattern of protein phosphorylation during germination depends on the method of spore activation. Autoactivation appears to involve activation of phospholipase $\mathrm{C}$ and inositol 1,4,5 triphosphate production while heat-activated germination does not (Lydan \& Cotter, 1995).

In summary, our results indicate: (i) higher cAMP levels in spores than in nascent amoebae, (ii) a transient cAMP increase precedes the decrease to low levels in amoebae during spore germination, (iii) inhibition of autoactivation with permeable cAMP agonists, (iv) spore cAMP levels are dynamic during dormancy and the primary target of the cAMP signal may be PKA (van Es et al., 1996). These results compel us to describe spore dormancy in D. discoideum as dynamic. Also, the stages of sporulation, establishment of dormancy, spore ageing and early germination (including deactivation) represent contiguous events that may be based on a common cAMP-dependent pathway (Cotter et al., 1992). Characterization of this pathway(s) and its sensitivity to other germination signals such as the autoinhibitor (discadenine) and autoactivator are essential to our understanding of post-sporulation events.

\section{ACKNOWLEDGEMENTS}

The authors thank Dr P. Devreotes for discussion and Drs M. Lydan, B. Mutus, D. Thomas and A. Warner for critically reading the manuscript, and $\mathrm{Mrs} J$. Durocher for assistance with its preparation. We thank Dr M. Veron for the culture of the $r d e \mathrm{C}$ mutant strain HTY217. Support was provided by research grant No. 0GP0000390 from the Natural Sciences and Engineering Research Council of Canada.

\section{REFERENCES}

Abe, K. \& Yangisawa, K. (1983). A new class of rapidly developing mutants in Dictyostelium discoideum; implications for cAMP metabolism and cell differentiation. Dev Biol 95, 200-210.

Abe, H., Uchiyama, M., Tanaka, Y. \& Saite, H. (1976). Structure of discadenine, a spore germination inhibitor form the cellular slime mold Dictyostelium discoideum. Tetrahedron Lett 42, 3807-3810.

Anjard, C., Pinaud, S., Kay, R. R. \& Reymond, C. D. (1992). Overexpression of the DdPK2 protein kinase causes rapid development and affects the intracellular CAMP pathway of Dictyostelium discoideum. Development 115, 785-790.

Botsford, J. L. \& Harman, J. G. (1992). Cyclic AMP in prokaryotes. Microbiol Rev 56, 100-122.

Chandrasekhar, A., Ennis, H. L. \& Soll, D. R. (1992). Biological and molecular correlates between induced dedifferentiation and spore germination in Dictyostelium. Development 116, 417-425.

Chang, W.-T., Thomason, P. A., Gross, J. D. \& Newell, P. C. (1998). Evidence that the RdeA protein is a component of a multistep phosphorelay modulating rate of development in Dictyostelium. EMBO J 17, 2809-2816.

Cotter, D. A. (1981). Spore activation. In The Fungal Spore, pp. 385-411. Edited by A. Turian \& H. R. Hohl. New York: Academic Press.

Cotter, D. A. \& Glaves, M. L. (1989). Temporal control of 
autoactivator synthesis and secretion during spontaneous spore germination in Dictyostelium discoideum. Arch Microbiol 152, 44-51.

Cotter, D. A. \& Raper, K. B. (1968). Factors affecting the rate of heat-induced spore germination in Dictyostelium discoideum. $J$ Bacteriol 96, 86-92.

Cotter, D. A., Satre, M. \& Klein, G. (1990). Germination of Dictyostelium discoideum spores: inhibition of the autoactivator pathway by vanadate. $J$ Gen Microbiol 136, 841-845.

Cotter, D. A., Sands, T. W., Virdy, K. J., North, M. J. \& Klein, G. (1992). Patterning of development in Dictyostelium discoideum: factors regulating growth, differentiation, spore dormancy and germination. Biochem Cell Biol 70, 892-919.

Cotter, D. A., Dunbar, A. J., Buconjic, S. D. \& Wheldrake, J. F. (1999). Ammonium phosphate in sori of Dictyostelium discoideum promotes spore dormancy by stimulation of the osmosensor ACG. Microbiology 145, 1891-1901.

Dahlberg, K. R. \& Cotter, D. A. (1978). Autoactivation of spore germination in mutant and wild-type strains of Dictyostelium discoideum. Microbios 23, 153-166.

van Es, S., Virdy, K. J., Pitt, G. S., Meima, M., Sands, T. W., Devreotes, P. N., Cotter, D. A. \& Schaap, P. (1996). Adenylyl cyclase $\mathrm{G}$, an osmosensor controlling germination of Dictyostelium spores. J Biol Chem 271, 23623-23625.

Firtel, R. A. (1996). Interacting signalling pathways controlling multicellular development in Dictyostelium. Curr Opin Genet Dev 6, 545-554.

Glaves, M. L. \& Cotter, D. A. (1989). Environmental parameters limiting spore germination in a spontaneously germinating mutant of Dictyostelium discoideum. Mycologia 81, 115-121.

Goldberg, N. D. \& O'Toole, A. G. (1971). Analysis of cyclic 3',5'adenosine monophosphate and cyclic 3',5'-guanosine monophosphate. In Methods of Biochemical Analysis, pp. 1-39. Edited by D. Glick. New York: Wiley Interscience.

Houslay, M. D. \& Milligan, G. (1997). Tailoring cAMP signalling responses through isoform multiplicity. Trends Biochem $S_{c i} 22$, 217-224.

Kay, R. R. (1989). Evidence that elevated intracellular cyclic AMP triggers spore maturation in Dictyostelium. Development 105, 753-759.

Kessin, R. H. (1977). Mutations causing rapid development of Dictyostelium discoideum. Cell 10, 703-708.

Kessin, R. H., Orlow, S., Shapiro, R. I. \& Franke, J. (1979). Binding of inhibitor alters kinetic and physical properties of extracellular cAMP phosphodiesterase EC 3.1.4.17 from Dictyostelium discoideum. Proc Natl Acad Sci USA 76, 5450-5454.

Kim, H.-J., Chang, W.-T., Meima, M., Gross, J. D. \& Schaap, P. (1998). A novel adenylyl cyclase detected in rapidly developing mutants of Dictyostelium. J Biol Chem 273, 30859-30862.

Kishi, Y., Clements, C., Mahadeo, D. C., Cotter, D. A. \& Sameshima, M. (1998). High levels of actin tyrosine phosphorylation: correlation with the dormant state of Dictyostelium spores. J Cell Sci 111, 2923-2932.

Klein, G., Cotter, D. A., Martin, J. B., Bof, M. \& Satre, M. (1988). Germination of Dictyostelium discoideum spores: a ${ }^{31} \mathrm{P}$ NMR analysis. Biochemistry 27, 8199-8203.

Kwong, L. \& Weeks, G. (1990). The effects of presumptive morphogens on prestalk and prespore gene expression in monolayers of Dictyostelium. Differentiation 44, 88-94.

Loomis, W. F. (1982). The Development of Dictyostelium discoideum. New York: Academic Press.
Loomis, W. F. (1998). Role of PKA in the timing of developmental events in Dictyostelium cells. Microbiol Mol Biol Rev 62, $684-694$.

Loomis, W. F., Shaulsky, G. \& Wang, N. (1997). Histidine kinases in signal transduction pathways in eukaryotes. J Cell Sci 110, 1141-1145.

Lydan, M. A. \& Cotter, D. A. (1995). The role of $\mathrm{Ca}^{2+}$ during spore germination in Dictyostelium: autoactivation is mediated by the mobilization of $\mathrm{Ca}^{2+}$ while amoebal emergence requires entry of external $\mathrm{Ca}^{2+}$. J Cell Sci 108, 1921-1930.

Lydan, M. A., Cotter, D. A. \& O'Day, D. H. (1994). Stage-specific changes in protein phosphorylation during spore germination in Dictyostelium: role of calmodulin. Biochem Biophys Res Commun 201, 430-435.

Maeda, M. (1992). Efficient induction of sporulation of Dictyostelium prespore cells by 8-bromo cAMP under both submerged and shaken culture conditions and involvement of protein kinase(s) in its action. Dev Growth Diff 34, 263-275.

Malkinson, A. M. \& Ashworth, J. M. (1973). Adenosine 3',5' cyclic monophosphate concentrations and phosphodiesterase activities during axenic growth and differentiation of cells of the cellular slime mould Dictyostelium discoideum. Biochem J 134, 311-319.

Nellen, E., Datta, S., Reymond, C., Siversten, A., Mann, S., Crowley, T. \& Firtel, R. A. (1987). Molecular biology in Dictyostelium: tools and applications. Methods Cell Biol 28, 67-100.

North, M. J. \& Cotter, D. A. (1984). Proteolytic enzyme activity during Dictyostelium discoideum spore germination Exp Mycol 8, 47-58.

Peters, D. J. M., Cammans, M., Smit, S., Spek, W., VanLookeren Campagne, M. M. \& Schaap, R. (1991). Control of cAMP-induced gene expression by divergent signal transduction pathways. Dev Genet 12, 25-34.

Pitt, G. S., Milona, N., Borleis, J., Lin, K. C., Reed, R. R. \& Devreotes, P. N. (1992). Structurally distinct and stage-specific adenylyl cyclase genes play different roles in Dictyostelium development. Cell 69, 305-315.

Riley, B. B. \& Barclay, S. L. (1990). Ammonia promotes accumulation of intracellular cyclic AMP in differentiating amoebae of Dictyostelium discoideum. Development 109, 715-722.

Riley, B. B., Jenson, B. R. \& Barclay, S. L. (1989). Conditions that elevate intracellular cyclic AMP levels promote spore formation in Dictyostelium. Differentiation 41, 5-13.

Schaap. P. (1991). Intercellular interactions during Dictyostelium development. In Microbial Cell-Cell Interaction, pp. 147-166. Edited by M. Dworkin. Washington: American Society for Microbiology.

Shaulsky, G., Escalante, R. \& Loomis, W. T. (1996). Developmental signal transduction pathways uncoded by genetic suppressors. Proc Natl Acad Sci USA 93, 15260-15265.

Shaulsky, G., Fuller, D. \& Loomis, W. F. (1998). A cAMP phosphodiesterase controls PKA dependent differentiation. Development 125, 691-699.

Simon, M., Pelegrini, O., Vernon, M. \& Kay, R. (1992). Mutation of protein kinase A causes heterochronic development of Dictyostelium. Nature 356, 171-172.

Thomason, P. A., Traynor, D., Cavet, G., Chang, W.-T., Harwood, A. J. \& Kay, R. R. (1998). An intersection of the cAMP/PKA and two component signal transduction systems in Dictyostelium. EMBO J 17, 2838-2845.

Van Haastert, P. J. M., Janssens, P. M. W. \& Erneux, C. (1991). 
Sensory transduction in eukaryotes: a comparison between Dictyostelium and vertebrate cells. Eur J Biochem 195, 289-303.

Wang, B. \& Kuspa, A. (1997). Dictyostelium development in the absence of cAMP. Science 277, 251-253.

Zinda, M. J. \& Singleton, C. K. (1998). The hybrid histidine kinase $d h k B$ regulates spore germination in Dictyostelium discoideum. Dev Biol 196, 171-183.

Received 5 November 1998; revised 6 April 1999; accepted 20 April 1999. 